\title{
A Comparative Study of Classifiers for Tumor Detection
}

\author{
Priya M. Jadhav \\ PG Student, Dept. of CSE \\ K.L.E. Institute of Technology \\ Hubli - 580030
}

\author{
Manu T. M. \\ Professor, Dept. of ECE \\ K.L.E. Institute of Technology \\ Hubli - 580030
}

\begin{abstract}
Image-processing is a demanding field that includes various applications such as CT-scan, angiography, MRI etc. MRI is the standard non invasive skill used for analyzing, diagnosing and treating the abnormal tissues. In the proposed method for improving the contrast we utilized enhancement techniques. For skull striping adaptive thresholding and morphological operations are being employed. For extraction of features we employed GLRLM. Further we applied some techniques such as linear-SVC, decision tree and SVM for classifying the brain MRI images. SVM provided effective and accurate results among all the classifiers.
\end{abstract}

\section{Keywords}

Magnetic Resonance Imaging, Gray-Level Run Length Matrix, Brain Tumor, Segmentation, Morphology, SVM Classifier.

\section{INTRODUCTION}

Brain is the intermediate core part of the human body that commands their nervous system. Brain commands many functions like heart, respiring, speaking, strolling, ability to think etc. Hence, it plays an important role in human nervous system.

Usually when cells grow old or get destructed, they die and new cells take their spot. This process might not be correct always. When the body will not be working properly due to inner defects of cells or organs, new cells originate, and thus the origination of new cells forms a mass of tissue which is actually called as growth of tumor [10]. Tumors can be of two types and those are Benign or Malignant brain tumors.

Benign or acute brain tumors do not accommodate cancer cells. As cells grow back rarely it is easy to eliminate these cells from the body. They have a very clear boundary. Cells present in benign tumors rarely occupy the tissues surrounding them. Usually these cells don't spread. Rarely i.e. $10 \%$ among $100 \%$, these tumors are life threatening.

Malignant or severe brain tumors accommodate cancer cells. As cells grow very fast it is very hard to eliminate these cells from the body. Cells present in malignant tumors quickly occupy the tissues surrounding them. As they don't have clear boundary, they cannot be identified and removed soon. Malignant tumors are mostly life threatening [8].

Indications generate when a tumor is pressed on a nerve or some part of the brain is got injured and also it mainly depend on its class, dimension and position. They might also be generated when there is no proper flow of blood in body specifically brain or some hurdles are jamming the flow of fluids. Early injuries or wounds don't get healed soon.

MRI is a skill used in radiology to get the detailed pictures of the areas and the physiological processes of the body with respect to health and disease. These scanners utilize strong magnetic fields and radio waves to produce images of the organs in the body [1]. MRI is a medical application of NMR i.e. nuclear magnetic resonance. It is also a choice of investigation in the preoperative and preoperative stages. It has wide range of applications in the medical stream and also assembles the pictures of neurological, cardiovascular, musculoskeletal, liver, gastro intestinal etc [12].

\subsection{Literature Survey}

(A. Sivaramakrishnan et al., 2015) have proposed a method for detecting the tumor by taking MRI-images as an input. Also employed principle-component-analysis for image rotting that is used to decrease the waveletcoefficient dimension. Segmentation is carried out by making use of FCM and histogram-equalization. Here FCM gives the highest accuracy on an average of $70 \%$.

(Riries Rulaningtyas et al., 1998) have proposed a method for detecting the tumor by taking MRI-images as an input. They utilized three different kinds of algorithms such as Prewitt, Robert and Sobel that detect the edges. For accessing gradients in both $\mathrm{x}$ and $\mathrm{y}$ axis we employed a set of $3 \times 3$ convolution masks. Since Sobel edge detection algorithm exhibits very little mean and standard-deviation value, it is ideally being used.

(Kiran Thapaliya et al., 2014) have proposed a method for detecting the tumor by MRI images as an input. Employed median filter for filtering of images. For the enhancement of intensity, first the morpho-gradient is calculated and later added to the filtered image. By making use of standard deviation and mean of the pictures threshold value is being calculated. For the extraction of tumors we employed flood fill algorithm and pixel replacement process

(Sahar Ghanavati et.al.,) have proposed a method for detecting the tumor by taking MRI-images as an input. Three different types of brain MRI modalities such as T1-weighted, T2-weighted, T1--GCA are being deployed in this work. For every image features such as intensity, shape, symmetry and texture are being extracted. Segmentation process is carried out here by making use of AdaBoost-classifier. AdaBoost-classifier is ideally used to elect the features and segment the tumor region. Here AdaBoost-classifier gives the highest accuracy on an average of $78 \%$. 
The remaining part of the paper is organized into three sections. Section II deals with methodology developed in this work. The experimental results are discussed in section III. The paper is concluded in section IV.

\section{METHODOLOGY}

MRI Images are collected from various sources such as Hospitals, Radiologists and from the Internet. The database contains MRI-scans which are in JPEG image-format. The number of used data for classification is of 20 normal brains. 24 Severe tumors and 12 Acute tumors. Totally there are of $56 \mathrm{MRI}$ images.

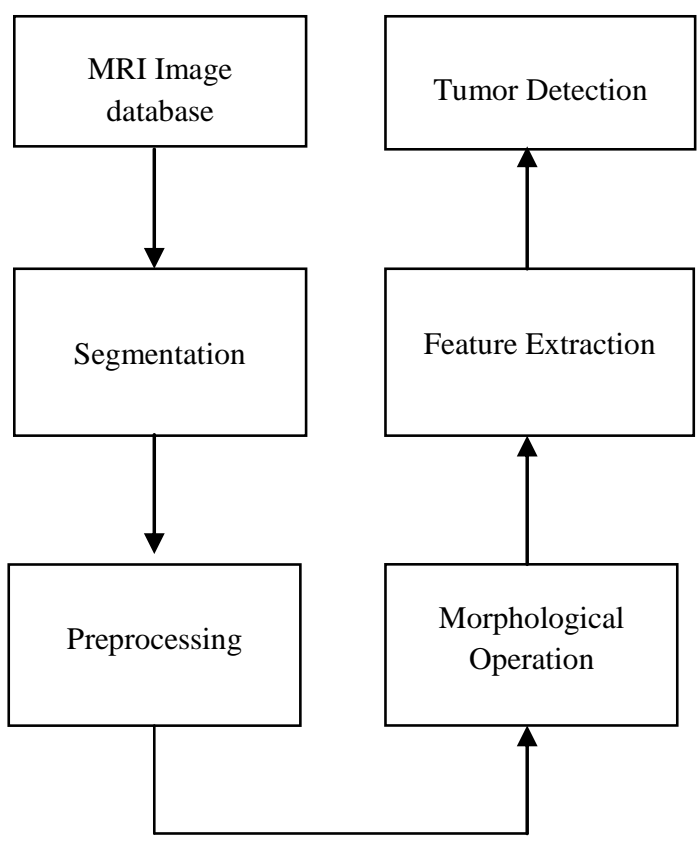

Figure 1: Flow Chart for Tumor Detection

\subsection{Pre-processing}

Pre-processing is a purpose of filtering image-data which in turn quells un-wanted distortions into features of that image that are important for further-processing [9]. Grayscale-conversion, histogram-equalization, image denoising, skull-striping and image enhancement are some of the operations pertained on pictures as part of preprocessing [3].

\subsubsection{Grayscale Conversion}

Gray-shading is obtained by contemplating the picture with a set of black-dots on a white back-ground / whitedots on a black back-ground, and the size of every single dot will determine the manifest-lightness of gray with surrounding place [2]. RGB components brightness level is symbolized as a no. from 0 to 255 in decimals / 00000000 to 11111111 in binary [7].

\subsubsection{Histogram Equalization}

Histogram-equalization is utilized to intensify the picture quality and a technique for adjusting image intensities to heighten the contrast [12]. When the picture is transformed to a better meaningful structure we can easily calculate the frequency w.r.t. each gray-value. The output obtained from histogram-equalization technique shows that the dissimilarity of an image is intensified [2].

\subsubsection{Image De-noising}

Image de-noising is a process with which we rebuild a signal from a noisy one. Technically it is termed as removing unwanted noise for replacing original-image. Various noise removal techniques such as spatial filtering, linear filters, mean filters, non-linear filters, median filter are used. While converting a picture i.e. RGB to grayscale, some kind of disturbance enters to the picture [5]. Through filtering-technique we can extract that disturbance / noise. From the value of pixel in the center with the neighboring pixels, disturbances like pepper, salt can be eradicated [3].

\subsubsection{Skull Striping}

Skull striping is termed as means of removing non-brain tissues such as fat, skin, osseous tissues, muscles, connective tissues and eyeball from the MR image of the brain. It authorizes the processing of brain images in more successful way and also upgrades the final result [4].

\subsubsection{Enhancement of an Image}

Whatever image we obtain may exhibit some imperfections like inferior contrast. Such imperfections lead to enormous effect on images. Contrast improvement skill will be taken into action if and only if there exist inferior contrast. In order to enhance the contrast, every single pixel of gray-level will be mounted [1].

\subsection{Segmentation Using Adaptive Thresholding}

A phenomenon to divide a digital picture in the form of multiple-chunks is referred as segmentation. The aim of segmenting a picture is relevant and very easy to examine. More exactly, Image-segmentation is the procedure of allocating label for every pixel in such a way that those pixels which exhibit similar label split specific optical features [1]. Effortless technique of Image-segmentation is referred as adaptive thresholding-method. This procedure depends on threshold-value which transforms a gray-scale picture to a binary picture [8]

\subsection{Morphological Operation}

Morphology is particularly termed as the interpretation of the characteristics such as appearance, fabric. Morphological-operations refer to reasoning transformation which depends on differentiating neighbor pixel w.r.t. design [4]. Morphological-operations performed habitually on binary-images and the pixel values are either $0 / 1$. Nearly all morphological-operations mainly pivot on binary-images [10].

\subsection{Feature Extraction}

Feature extraction is a process of transferring data into group of features [6]. In machine learning it is termed as pattern recognition while in image processing its termed as feature extraction [4]. If we select the extracted features in a proper way, those feature set will perform the desired task. These features are further useful in classifying the images [11].

\section{EXPERIMENTAL RESULTS}

Tumor identification is an experiment being fetched in PYTHON by making use of several operations. The snapshots of different levels are shown below. Extraction of tumor is elaborated in every level. The proposed methodology is implemented successfully. 


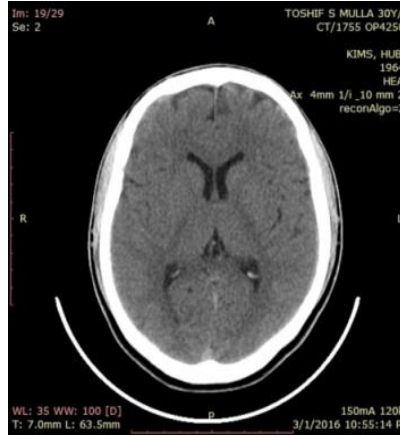

(a)

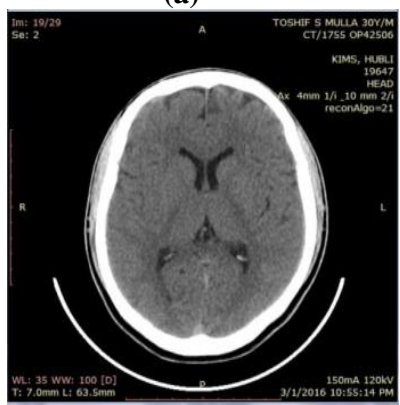

(c)

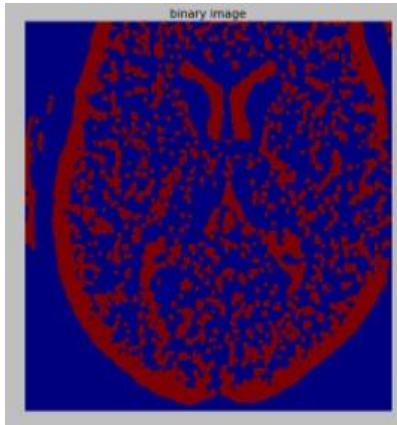

(e)

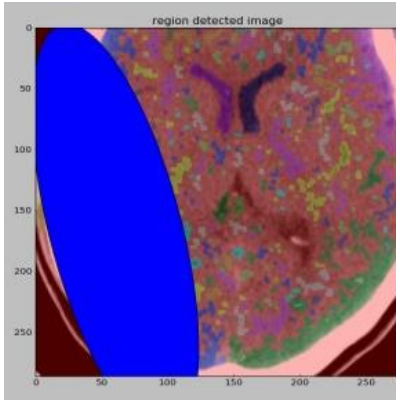

(g)

Figure 2: Brain MRI Images (a) Image Acquired, (b)

Grayscale Image, (c) Contrast Stretched Image, (d) Main Brain Image, (e) Binary Image, (f) Labeled Image and (g) Region Detected Image

The performance measures used in machine learning are [2]:

$>$ Accuracy / System Reliability $=(\mathrm{ARV}+\mathrm{APV}) /$ $(\mathrm{ARV}+\mathrm{IPV}+\mathrm{IRV}+\mathrm{APV})$

$>$ Sensitivity / Recall / Accurate Practical Value = APV / (IRV + APV)

$>$ Specificity / Accurate Refusal Value $=$ IPV / (ARV + IPV)

$>$ Precision $=\mathrm{APV} /(\mathrm{IPV}+\mathrm{APV})$

The output images are shown in Figure 3 through Figure 5.

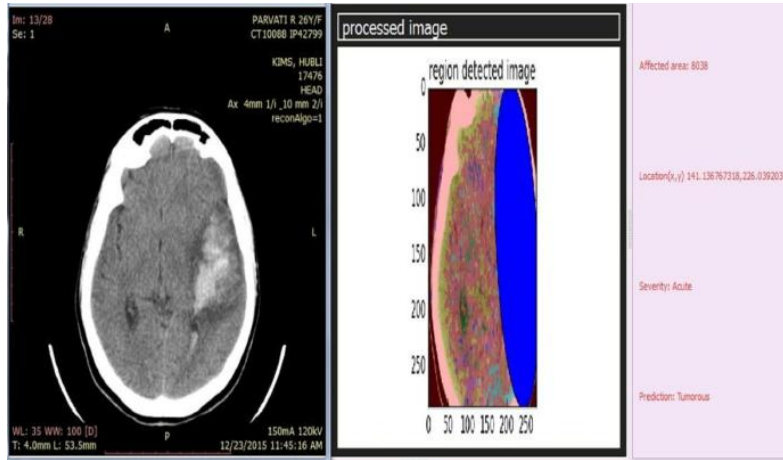

Figure 3: Output of Acute Tumor Affected Brain

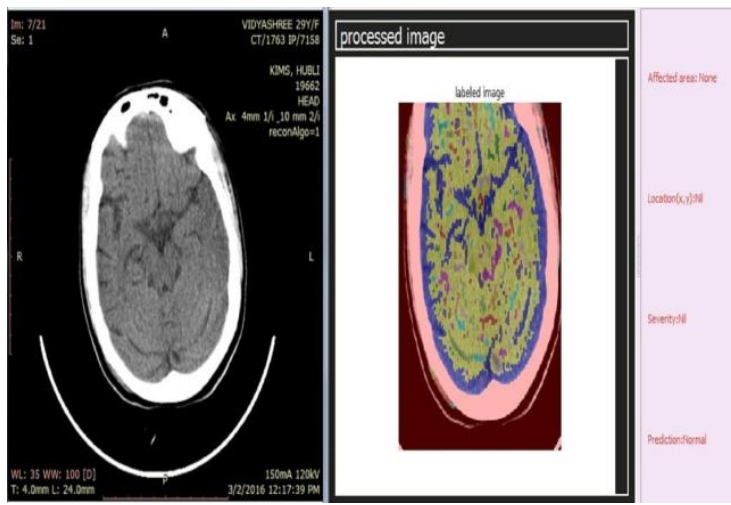

Figure 4: Output of Non-Tumor Affected Brain

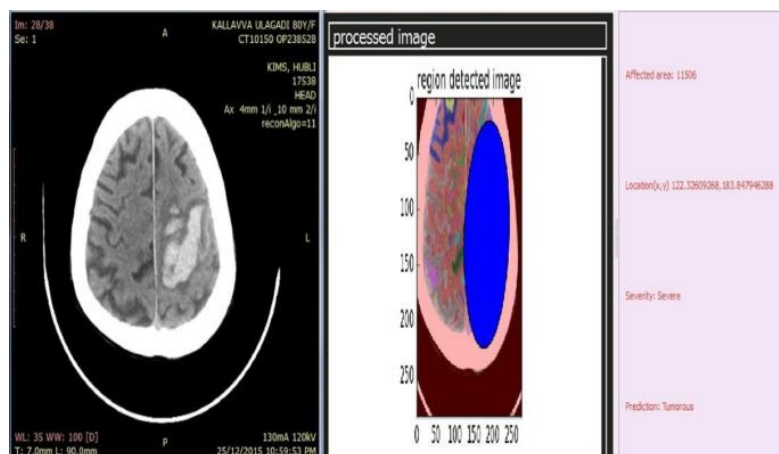

Figure 5: Output of Severe Tumor Affected Brain

The ROC Curve and the confusion matrix with the calculation of accuracy of the images using different classifiers are shown in Figure 6 through Figure 14. The accuracy score is shown in Figure 15. The performance of classifier is shown in Table 1.

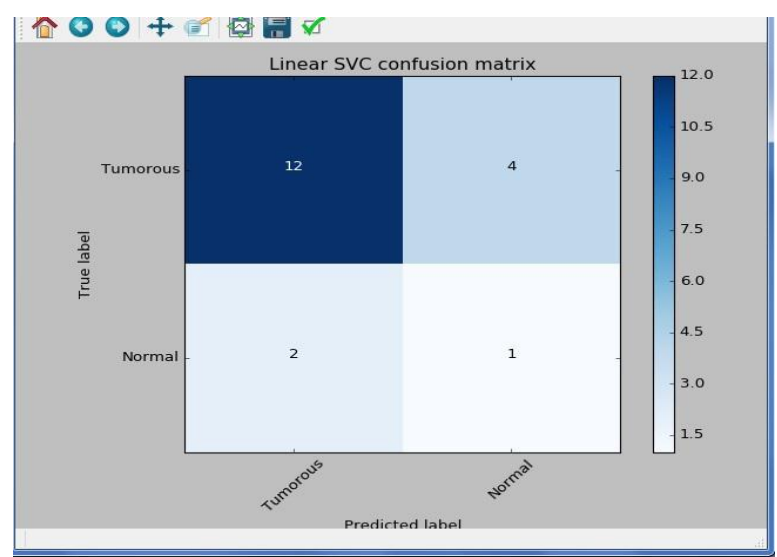

Figure 6: Confusion matrix for Linear SVC. 


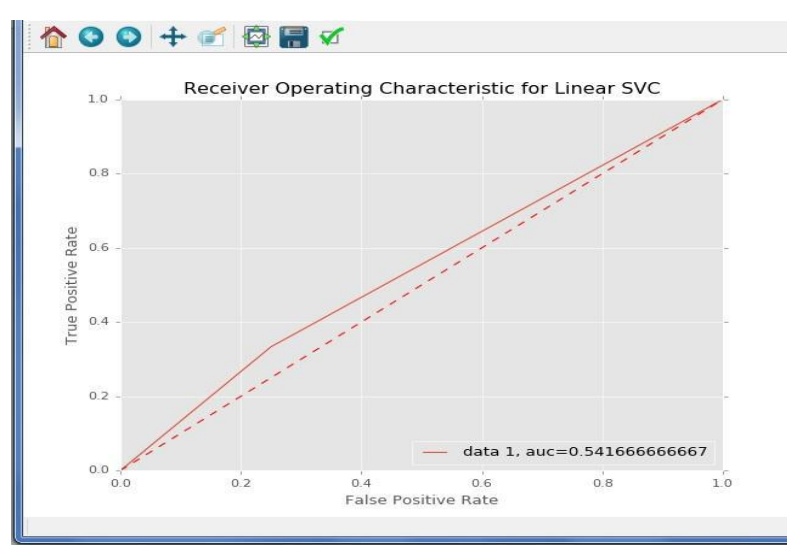

Figure 7: ROC Curve for Linear SVC.

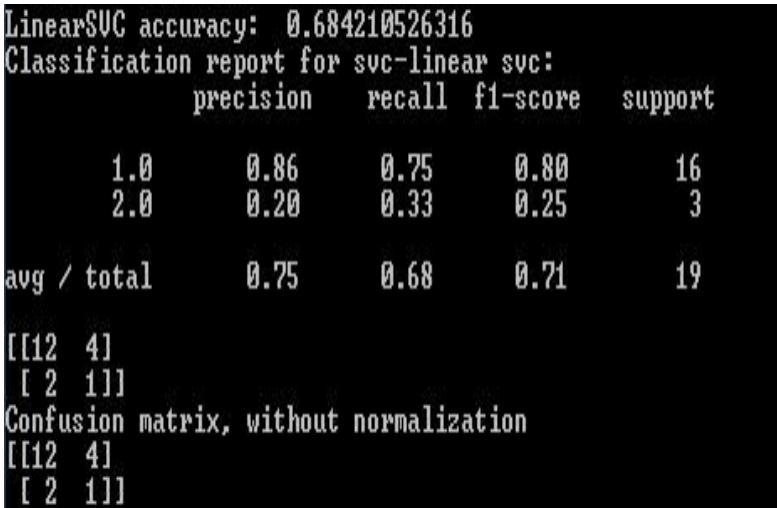

Figure 8: Calculation of Accuracy from the Linear SVC.

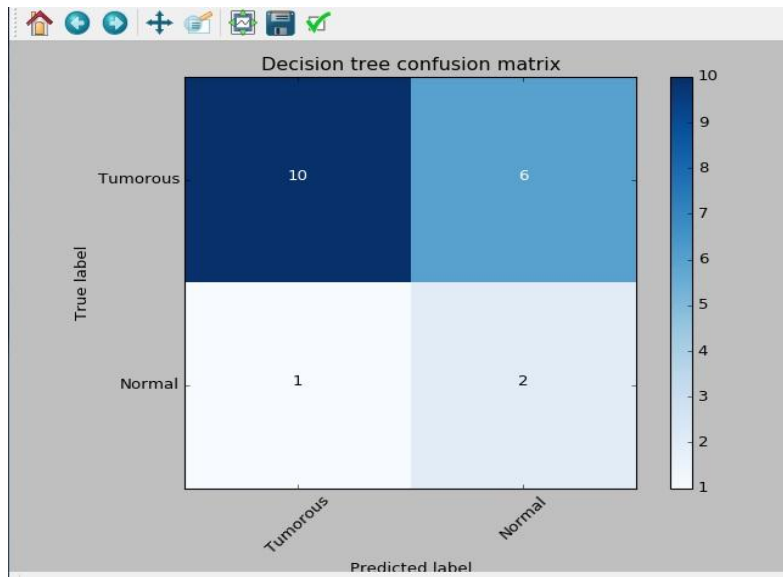

Figure 9: Confusion matrix for Decision Tree.

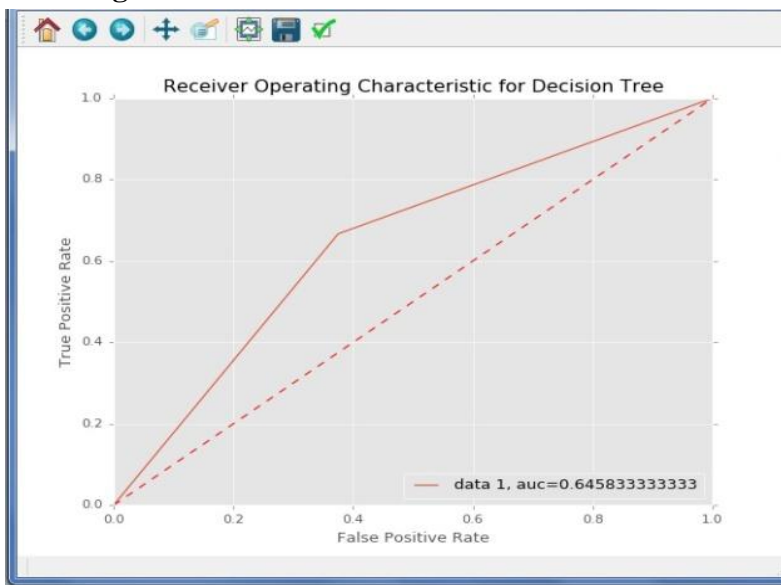

Figure 10: ROC Curve for Decision Tree.

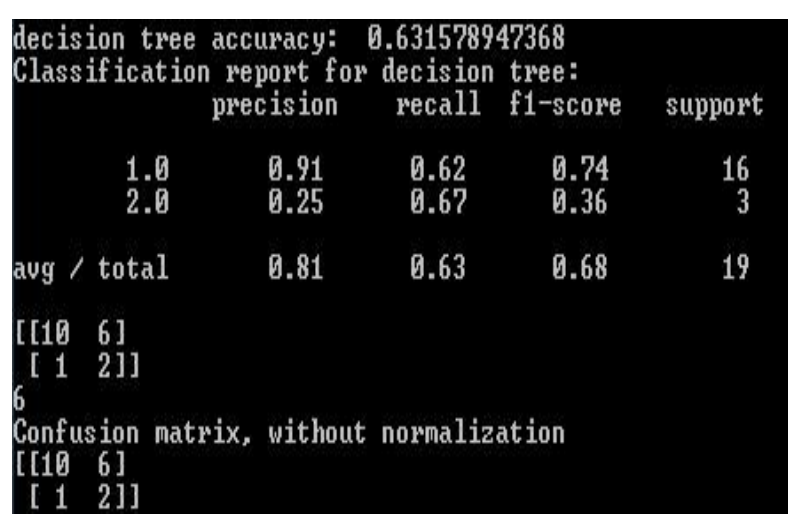

Figure 11: Calculation of Accuracy from the Decision Tree

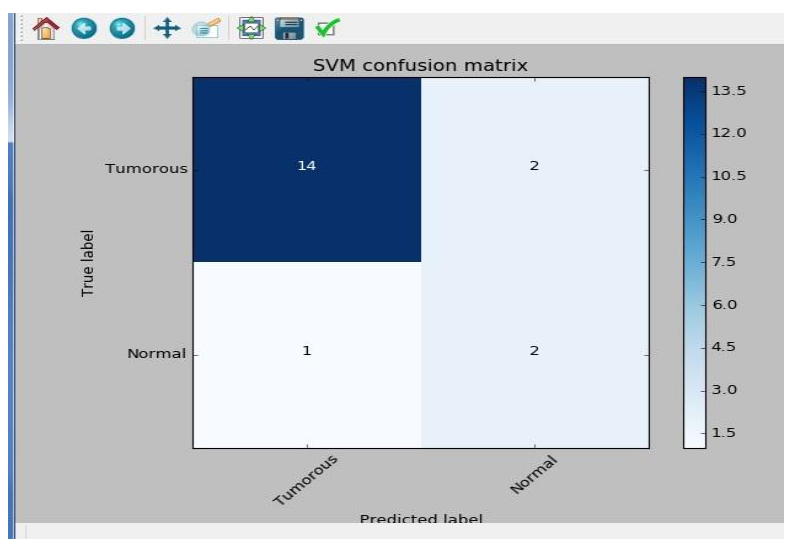

Figure 12: Confusion matrix for SVM Classifier.

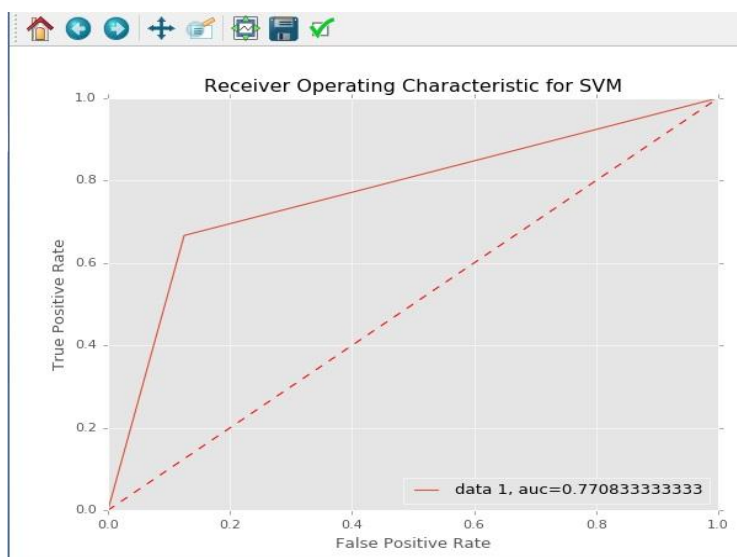

Figure 13: ROC Curve for SVM Classifier.

SUM accuracy: $\quad 0.842105263158$

Classification report for SUM:

$\begin{array}{rrrrr} & \text { precision } & \text { recall } & \text { f1-score } & \text { suppor } \\ 1.0 & 0.93 & 0.88 & 0.90 & 16 \\ 2.0 & 0.50 & 0.67 & 0.57 & 3 \\ \text { aug / total } & 0.86 & 0.84 & 0.85 & 19\end{array}$

[ [14 2]

[ 112$]]$

Confusion matrix, without normalization

[ [ [14 2]

$\left[\begin{array}{ll}1 & 2\end{array}\right]$

Figure 14: Calculation of Accuracy from the SVM 
Table 1: Performance Analysis Chart for Different Classifiers

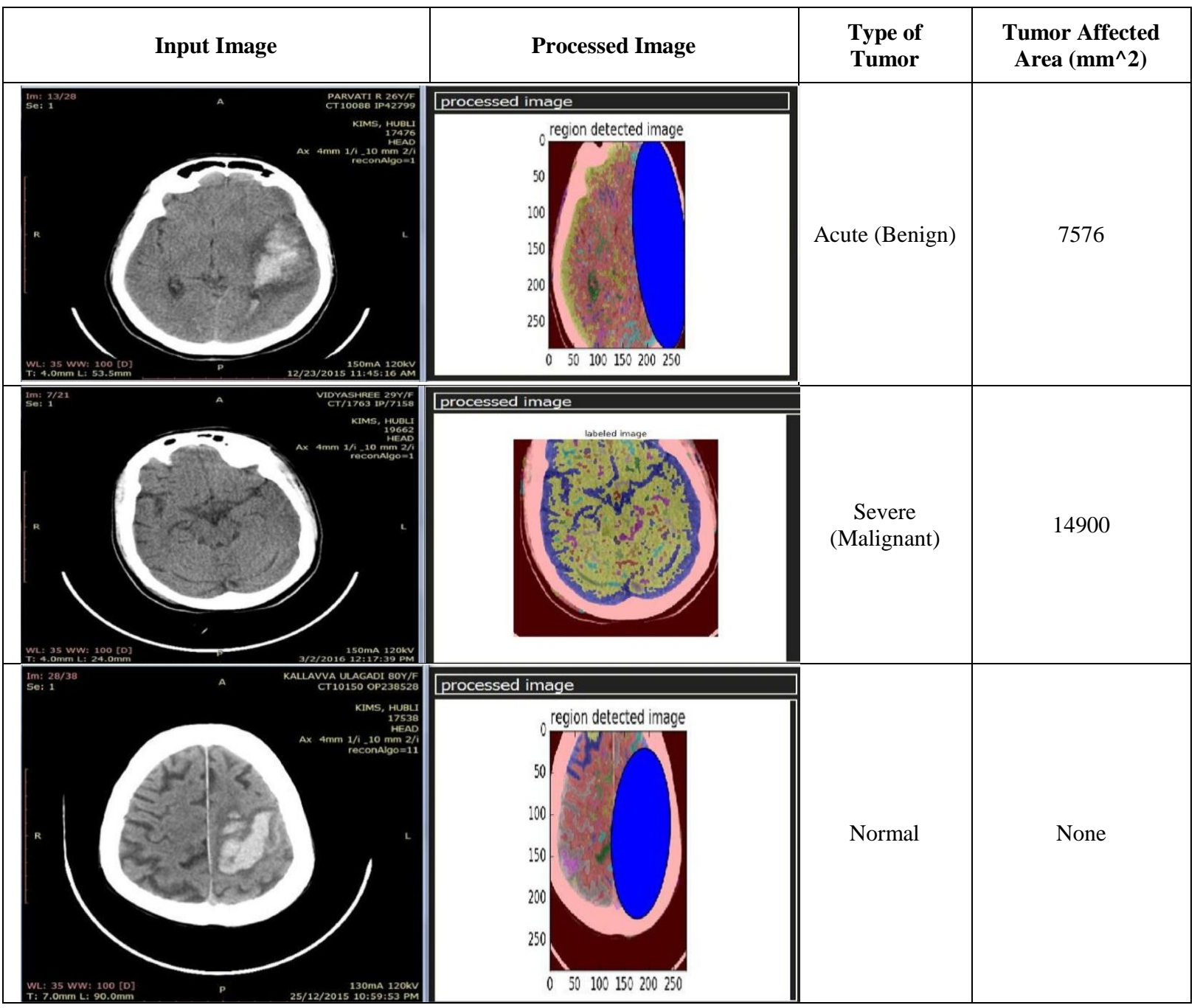

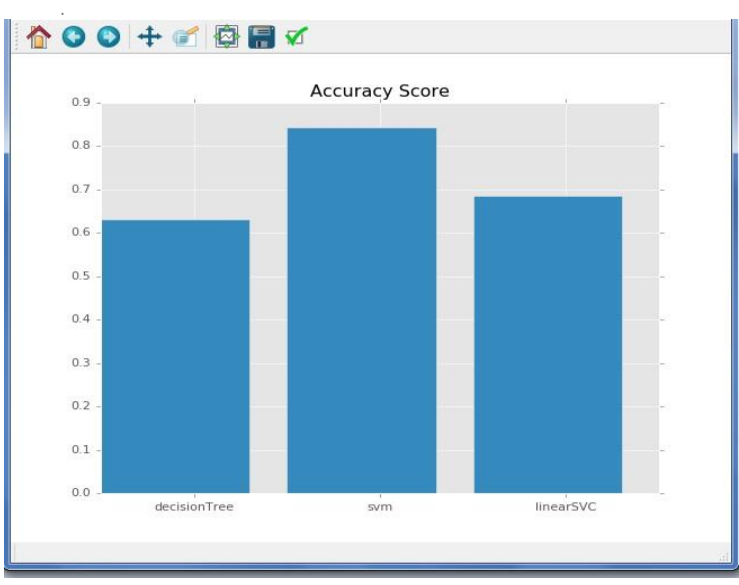

Figure 15: Output for Accuracy Score

\section{CONCLUSION}

The advanced methodology in MR images is an effective form to locate and arrange brain tumor. Pre-processing of MR images was done by employing gray-scaling, histogram equalization and filtering techniques. It is highly based on mathematical morphology and thresholding. Thresholding process was utilized to work on desired area or region of an image and also to convert an image into binary form.

Outcomes are presented images with brain tumor and extrication of affected fragment of the brain. The SVM technique used for classification provided more accurate results compared to Linear SVC and Decision Tree algorithm. Proposed technique is invariant in terms of intensity, size and shape. Oftenly it marks the position of the tumor so that visualization becomes easy and convenient. The methodology aims more perfection rather than misconception.

In future, if one expands the proposed algorithm by segmenting several images with several characteristics which allows us in classifying different types of tumors. By applying advanced classification algorithms and clustering algorithms one can obtain better efficiency. By employing some new skills one can reduce the computation time. In order to get accurate results one can analyze the texture features which plays an important role. 


\section{REFERENCES}

[1]. A S Basavaraj S. Anami, and Prakash H. Unki. "Multilevel thresholding and fractal analysis based approach for classification of brain MRI images into tumour and non-tumour", International Journal of Medical Engineering and Informatics, 2016ivaramakrishnan and Manoj Kowar "Brain Tumor Detection and Segmentation Using Histogram Thresholding", International Journal of Engineering and Advanced Technology, Volume 1, Issue 4, April 2012.

[2]. Riries R and J M Jernigan "Automatic Segmentation of Cerebral MR Images using Artificial Neural Network", IEEE Transactions on Nuclear Science, Volume 4, 1998.

[3]. K.Thapaliya and Goo-Rak Kwon "Morphological Operations to Segment a Tumor from A Magnetic Resonance Image", Journal of Information and Communication Convergence Engineering, pp. 60-65, March 2014.

[4]. Sahar Ghanavati and Junning Li "Automatic Brain Tumor Detection In Magnetic Resonance Images", International Conference on Medical Imaging, University of Torronto, Canada.

[5]. Shrutika Santosh Hunnur, Akshata Raut and Swati Kulkarni. "Implementation of image processing for detection of brain tumors", 2017 International Conference on Intelligent Computing and Control Systems (ICICCS), 2017

[6]. Natarajan, P., N Krishnan, Natasha Sandeep Kenkre, Shraiya Nancy, and Bhuvanesh Pratap Singh. "Tumor detection using threshold operation in MRI brain images", 2012 IEEE International Conference on Computational Intelligence and Computing Research, 2012.

[7]. K M Iftekar and A E Lashkar "A Neural Network Based Method For Brain Abnormality Detection In MR Images Using Gabor Wavelets", International Journal of Computer Applications, Volume 4, 2010.

[8]. Pavel Dvorak and J Subhashini "An Efficient Brain Tumor Detection Methodology Using K-Means Clustering Algorithm", International Conference on Communication and Signal Processing, 2013, India.

[9]. Nailah Afshan, Shaima Qureshi and Syed Mujtiba Hussain. "Comparative study of tumor detection algorithms", International Conference on Medical Imaging, m-Health and Emerging Communication Systems, 2014.

[10].Yang Liu, Jinzhu Yang, Dazhe Zhao and Jiren Liu. "A method of pulmonary nodule detection utilizing multiple support vector machines", International Conference on Computer Application and System Modeling, 2010.

[11].Abd El Kader Isselmou, Shuai Zhang, Guizhi Xu. "A Novel Approach for Brain Tumor Detection Using MRI Images", Journal of Biomedical Science and Engineering, 2016.

[12].Basavaraj S. Anami, and Prakash H. Unki. "Multilevel thresholding and fractal analysis based approach for classification of brain MRI images into tumour and non-tumour", International Journal of Medical Engineering and Informatics, 2016 